\title{
An Intersectional Viral-Genetic Method for Fluorescent Tracing of Axon Collaterals Reveals Details of Noradrenergic Locus Coeruleus Structure
}

\author{
Nicholas W. Plummer, ${ }^{{ }^{\star}}$ (DDaniel J. Chandler, ${ }^{2^{\star}}$ Jeanne M. Powell, ${ }^{1}$ Erica L. Scappini, ${ }^{3}$ \\ Barry D. Waterhouse, ${ }^{2}$ and 1 Patricia Jensen ${ }^{1}$
}

https://doi.org/10.1523/ENEURO.0010-20.2020

${ }^{1}$ Neurobiology Laboratory, National Institute of Environmental Health Sciences, National Institutes of Health, Department of Health and Human Services, Research Triangle Park, NC 27709, ${ }^{2}$ Department of Cell Biology and Neuroscience, Rowan University School of Osteopathic Medicine, Stratford, NJ 08084, and ${ }^{3}$ Signal Transduction Laboratory, National Institute of Environmental Health Sciences, National Institutes of Health, Department of Health and Human Services, Research Triangle Park, NC 27709

\begin{abstract}
Understanding the function of broadly projecting neurons depends on comprehensive knowledge of the distribution and targets of their axon collaterals. While retrograde tracers and, more recently, retrograde viral vectors have been used to identify efferent projections, they have limited ability to reveal the full pattern of axon collaterals from complex, heterogeneous neuronal populations. Here we describe TrAC (tracing axon collaterals), an intersectional recombinase-based viral-genetic strategy that allows simultaneous visualization of axons from a genetically defined neuronal population and a projection-based subpopulation. To test this new method, we have applied TrAC to analysis of locus coeruleus norepinephrine (LC-NE)-containing neurons projecting to medial prefrontal cortex (mPFC) and primary motor cortex (M1) in laboratory mice. TrAC allowed us to label each projection-based LC-NE subpopulation, together with all remaining LC-NE neurons, in isolation from other noradrenergic populations. This analysis revealed mPFC-projecting and M1-projecting LC-NE subpopulations differ from each other and from the LC as a whole in their patterns of axon collateralization. Thus, TrAC complements and extends existing axon tracing methods by permitting analyses that have not previously been possible with complex genetically defined neuronal populations.
\end{abstract}

Key words: axon tracing; CAV2; locus coeruleus; noradrenergic; norepinephrine; recombinase

\section{Significance Statement}

We have developed a new method for mapping axon collaterals of genetically defined neuronal subtypes. TrAC (tracing axon collaterals) uses an intersectional genetic strategy to define a cell population of interest, and a retrograde viral construct to label a subpopulation based on its axonal projections. This method has three major benefits: (1) only one viral injection is required for labeling, (2) axons from a projection-based subpopulation can be visualized together with a broader genetically defined population, and (3) the cell population of interest can be defined by transient developmental genetic information. Our proof-of-principle analysis of noradrenergic locus coeruleus (LC) projections to the forebrain extends previous analyses and reveals new details of this complex system.

Received January 9, 2020; accepted April 27, 2020; First published April 30, 2020.

The authors declare no competing financial interests.
Author contributions: N.W.P., D.J.C., B.D.W., and P.J. designed research; N.W.P., D.J.C., J.M.P., and E.L.S. performed research; N.W.P., D.J.C., J.M.P., E.L.S., B.D.W., and P.J. analyzed data; N.W.P., D.J.C., B.D.W., and P.J. wrote the paper. 


\section{Introduction}

Fundamental to our understanding of neural circuit dynamics and function is comprehensive knowledge of the regions of the CNS innervated by neurochemically identified cell types. While conventional anatomic tract tracing methods have long been used to identify the afferent inputs and efferent projections of neuronal populations, these traditional approaches have proven impractical for revealing the full distribution of axon collaterals from a broadly projecting pool of neurons. One such brain region is the noradrenergic nucleus locus coeruleus (LC), a brainstem nucleus which provides norepinephrine (NE) to virtually the entire CNS. Early investigations into LC anatomy using conventional tracing methods suggested that its cells possess highly ramified axons with the potential to innervate broad regions of the CNS without regard for terminal field function (Bloom et al., 1971; Foote et al., 1980, 1983; Aston-Jones et al., 1986; Loughlin et al., 1986). Because of this apparent lack of functional organization, the LC has long been considered a homogeneous entity with neurons that release NE globally, thus promoting uniform actions throughout the brain and spinal cord simultaneously.

More recent evidence suggests that individual LC neurons innervate terminal fields in a more discrete and orderly fashion than originally thought (Simpson et al., 1997; Howorth et al., 2009; Chandler et al., 2014, 2019; Uematsu et al., 2015, 2017; Bellesi et al., 2016; Li et al., 2016; Hirschberg et al., 2017; McCall et al., 2017; Plummer et al., 2017). For example, it has been shown that LC cells projecting to medial prefrontal cortex (mPFC) are anatomically, molecularly, and electrophysiologically distinct from those that project to primary motor cortex (M1; Chandler et al., 2013, 2014). Additionally, injection of retrograde tracers into functionally related terminal fields (e.g., structures along the ascending somatosensory pathway) results in a higher percentage of double-labeled LC-NE neurons than when tracers are injected into functionally unrelated terminal fields (Simpson et al., 1997).

Based on these findings, there is renewed interest in identifying the collateral projections of the LC-NE system. Several laboratories have attempted to define the

This work was supported by the Intramural Research Program of the National Institutes of Health, National Institute of Environmental Health Sciences Grant ZIA-ES-102805 (to P.J.), and by the Extramural Research Program of the National Institutes of Health, National Institute of Mental Health Grant 1R01MH101178-01A1 (to B.D.W.)

${ }^{\star}$ N.W.P. and D.J.C. contributed equally to this work.

Acknowledgements: We thank Eric J. Kremer (Institut de Génétique Moléculaire de Montpellier) for providing the Cav2-Cre virus. We also thank the National Institute of Environmental Health Sciences (NIEHS) Comparative Medicine Branch and the NIEHS Fluorescence Microscopy and Imaging Center for valuable support.

Correspondence should be addressed to Patricia Jensen at patricia. jensen@nih.gov or Barry D. Waterhouse at waterhouse@rowan.edu.

https://doi.org/10.1523/ENEURO.0010-20.2020

Copyright (C) 2020 Plummer et al.

This is an open-access article distributed under the terms of the Creative Commons Attribution 4.0 International license, which permits unrestricted use, distribution and reproduction in any medium provided that the original work is properly attributed. organizational principles of this nucleus using a variety of novel approaches that have yielded conflicting results. While some have provided evidence for a modularly organized LC with subpopulations of neurons possessing limited axonal arborization confined to a small number of terminal fields (Chandler et al., 2013, 2014; Kebschull et al., 2016; Hirschberg et al., 2017; Uematsu et al., 2017), others have reported highly collateralized LC axons that innervate many functionally unrelated efferent targets (Schwarz et al., 2015). In order to resolve these discrepancies and gain a better understanding of the functional circuit-level organization of the LC, it is necessary to develop and apply methods that can faithfully identify the CNS regions that receive collateral projections from LCNE neurons projecting to a defined target. In a system this complex, with other brainstem noradrenergic neuron populations projecting to many of the same target sites, a method is required that can account for all LC-NE neurons without labeling any other NE neuron populations.

Toward this goal, we developed a methodology termed TrAC (tracing axon collaterals) that combines intersectional recombinase-based genetics with retrograde viral delivery of a recombinase to label axon collaterals from genetically defined cell types. Here, we describe TrAC and its application to reveal collateral networks of LC-NE neurons projecting to $\mathrm{mPFC}$ or $\mathrm{M} 1$, demonstrating the utility of this approach for dissecting the anatomy of complex, neurochemically identified neuronal populations. We found that retrograde viral delivery of a recombinase into either terminal field region produced similar numbers and distributions of retrogradely labeled LC neurons, but the patterns of axon collateralization were not identical. The labeled neurons had dense projections to the site of injection, with widespread, albeit sparse, collateral axons present in other cortical and subcortical targets. These findings are in general agreement with many other studies, suggesting that the LC-NE efferent pathway is functionally ordered and modular in design.

\section{Materials and Methods}

\section{Animals}

This study was performed in accordance with the recommendations in the Guide for the Care and Use of Laboratory animals of the National Institutes of Health. The protocols were approved by the Animal Care and Use Committees (ACUC) of the National Institute of Environmental Sciences and Rowan University.

To fluorescently label LC-NE neurons with tdTomato, B6.Cg-Gt(ROSA)26Sor tm1.1(CAG-tdTomato,-EGFP)Pjen mice (RC::RFLTG; The Jackson Laboratory stock no. 026930; Plummer et al., 2015) were intercrossed with B6.129En1 ${ }^{\text {tm1.1(dreo)Pjen }}$ (En1 ${ }^{\text {Dre }}$; The Jackson Laboratory Stock no. 033953; Plummer et al., 2016) and B6;129-Dbh ${ }^{\text {tm1(flpo)Pjen }}$ mice $\left(D b h^{\text {Flpo; }}\right.$ The Jackson Laboratory stock no. 033952; Robertson et al., 2013), generating triple heterozygotes. To subsequently label subpopulations of those LC-NE neurons with EGFP based on their axonal projection pattern, En $1^{\text {Dre }}$; Dbh ${ }^{\text {Flpo; }}$;C::RFLTG triple heterozygotes were stereotaxically injected in MPFC or M1 with 
the CAV2-Cre canine adenoviral vector (Hnasko et al., 2006).

\section{Surgery}

Male and female mice were deeply anesthetized through isoflurane inhalation (4\%) and placed in a stereotaxic frame. Isoflurane concentration was decreased to $2.5 \%$ after reaching a surgical plane of anesthesia. Body temperature was monitored and controlled throughout the surgical procedure. Surgery was performed in flat skull orientation such that bregma and $\lambda$ were level. The dorsoventral coordinate position of each was measured, and if they were found to be $>0.1 \mathrm{~mm}$ apart, the position of the nose cone was adjusted until they were level. Stereotaxic coordinates for viral infusions were as follows, in $\mathrm{mm}$, from bregma: mPFC: $\mathrm{AP}+1.95, \mathrm{ML}+0.9$, DV -2.5 at $15^{\circ}$ from the brain surface; M1: AP +0.26 , ML +1.2 , DV -0.63 from the brain surface. All infusions were made into the left hemisphere. After drilling a small craniotomy above either region, a 31-gauge Hamilton 1- $\mu$ l Neuros syringe (part \#7001) mounted on a World Precision Instruments stereotaxic syringe pump (model UMP3) was gradually lowered into the brain; $0.2-\mu$ l CAV2CMV-Cre $(5.6 \times 1012 \mathrm{pfu} / \mathrm{ml})$ was injected into either region at a flow rate of $0.5 \mu \mathrm{l} / \mathrm{min}$ using a World Precision Instruments Micro4 MicroSyringe Pump Controller. The needle remained in place undisturbed for $10 \mathrm{~min}$ on completion of the injection before being gradually withdrawn. Craniotomies were then filled with sterile bone wax and the incision was closed with wound clips. After surgery was complete, mice were returned to their home cages.

\section{Tissue collection}

Six weeks after viral injection, mice were anesthetized with sodium pentobarbital and transcardially perfused with 4\% paraformaldehyde (PFA) in $0.01 \mathrm{M}$ PBS. Dissected brains were postfixed by immersion in 4\% PFA at $4^{\circ} \mathrm{C}$. Brains to be sectioned were marked on the uninjected hemisphere with black TMD Tissue Marking Dye (General Data Company), equilibrated in $30 \%$ sucrose in PBS at $4^{\circ} \mathrm{C}$, and embedded in Tissue Freezing Medium (General Data Company). Free-floating 40- $\mu \mathrm{m}$ sections were collected in PBS and then stored at $-80^{\circ} \mathrm{C}$ in $30 \%$ sucrose/30\% ethylene glycol in PBS. For the passive clarity technique (PACT; Yang et al., 2014), 3-mm-thick slices containing the LC were cut using a stainless-steel coronal brain matrix (Zivic Instruments), and the remaining forebrain was embedded for sectioning as described above. PACT tissue clearing was performed as previously described (Plummer et al., 2015, 2017).

\section{Immunohistochemistry}

We used immunofluorescent labeling to enhance signal from fluorescent proteins in fixed tissue. For simultaneous detection of virally transduced (EGFP-labeled) and nontransduced (tdTomato-labeled) LC-NE axons, a chicken anti-GFP antibody (1:10,000; catalog \#ab13970, Abcam) was used in conjunction with Alexa Fluor 488 goat antichicken secondary antibody (1:1000; catalog \#A11039,
Thermo Fisher Scientific), and a rabbit anti-dsRed antibody (1:500; catalog \#632496, Clontech Laboratories) was used with Alexa Fluor 568 goat anti-rabbit secondary antibody (1:1000; catalog \#A11036, Thermo Fisher Scientific). Mouse monoclonal anti-NET antibody (1:1000; clone NET-05; catalog \#1447-NET, PhosphoSolutions; Matthies et al., 2009) was used with Alexa Fluor 633 goat anti-mouse secondary antibody (1:1000; catalog \#A21052, Thermo Fisher Scientific). Tissue was incubated with primary antibodies for $2 \mathrm{~d}$ at $4^{\circ} \mathrm{C}$ and with secondary antibodies for $2 \mathrm{~h}$ at room temperature. Following antibody incubations, sections were incubated for $1 \mathrm{~h}$ in a 1:50 dilution of Neurotrace 435/455 Blue Fluorescent Nissl Stain (Thermo Fisher Scientific) for detection of neuronal cell bodies.

For immunofluorescent labeling after PACT tissue clearing, we used chicken anti-GFP (1:1000) and rabbit anti-dsRed (1:500) primary antibodies, followed by Alexa Fluor 488 donkey anti-chicken $F(a b$ ')2 fragments (1:500; catalog \#703-546-155, Jackson ImmunoResearch) and Alexa Fluor 568 donkey anti-rabbit $F\left(a b^{\prime}\right) 2$ fragments (1:500; catalog \#Ab175694, Abcam). Incubations were performed at room temperature for $6 \mathrm{~d}$, with antibody replaced by fresh solution after $3 \mathrm{~d}$.

\section{Digital image collection}

LC-NE neurons in PACT-cleared tissue or $40-\mu \mathrm{m} \mathrm{sec-}$ tions were imaged on an LSM 780 or 880 inverted confocal microscope (Carl Zeiss Microscopy), using an EC Plan-Neuofluar $10 \times / 0.30 \mathrm{M} 27$ objective. For imaging axonal fibers, z-stack images through the full thickness of each $40-\mu \mathrm{m}$ section were collected on an LSM 880 confocal microscope using a PLAN Apochromat $20 \times 10.8$ M27 or Plan Aprochromat $40 \times / 1.3$ Oil M27 objective. Alexa Fluor 633 fluorescence was excited with a 633-nm laser (pinhole setting 1 airy unit), and fluorescent emission was collected with a 640- to 758-nm filter. Alexa Fluor 568 fluorescence was excited with a 561-nm laser and collected with a 571- to 633-nm filter. Alexa Fluor 488 was excited with a 488-nm laser and collected with a 491- to 562-nm filter, and also with a 571- to 633-nm filter for an autofluorescence-only channel to permit DEFiNE image processing (Powell et al., 2019). Blue Nissl fluorescence was excited with a 405-nm laser and collected with a 415- to 47-nm filter. For fiber quantification, each anatomic region was imaged from at least three sections. The investigator was blind to labeled fibers during selection of the regions to be imaged. Location was determined by external marking with Tissue Marking Dye (see above, Tissue collection) and by anatomy, as revealed by labeling with Nissl stain, compared with a mouse brain atlas (Paxinos and Franklin, 2013).

\section{Image processing and quantification of LC neurons and axons}

LC neurons were quantified from a total of four mice for each injection site (from 40- $\mu \mathrm{m}$ sections, $n=2 \mathrm{mPFC}$ injections and $n=1 \mathrm{M} 1$ injection; and from $40-\mu \mathrm{m}$ "virtual" sections of PACT-cleared tissue, $n=2$ mPFC injections 
and $n=3 \mathrm{M} 1$ injections). For counting LC-NE neurons in $40-\mu \mathrm{m}$ sections, $\mathrm{z}$-stack images were collected from every fourth section through the full rostrocaudal extent of the fluorescently labeled LC. Maximum-intensity projections (MIPs) were imported into FIJl software (Schindelin et al., 2012) for counting. Images collected from tissue cleared by PACT were viewed with Imaris software (Bitplane) for three-dimensional rendering of the entire region and collection of $40-\mu \mathrm{m}$ virtual sections using the Ortho Slicer function. Virtual sections spaced $160 \mu \mathrm{m}$ apart to match the sectioned tissue were imported into FIJI for cell counting.

For axon quantification, z-stack images collected with the $20 \times$ objective were first cropped to the center six zslices to ensure that the same volume of tissue would be compared in all images and to exclude autofluorescent particles such as dust or secondary antibody conglomerates lying between the tissue and coverslip; 400 $\times 400 \mu \mathrm{m}$ areas from larger images were selected for quantification, again using Nissl stain for identification of anatomic regions. This fixed size was chosen to maximize the volume quantified while remaining within the boundaries of regions defined by the mouse brain atlas (Paxinos and Franklin, 2013). To minimize the effects of possible variation in axon density within an anatomic brain region, axons were quantified in three sections from each region.

Before quantification, images were processed using the DEFiNE macro for FIJI (Powell et al., 2019) to remove autofluorescence and other fluorescent artifacts which would affect fiber density measurements. Images used as illustrations in figures were further processed only by adjusting brightness and contrast across the entire image. After conversion of the processed z-stack to a MIP, the DEFiNE Quantify Fibers function (Powell et al., 2019) was used to determine the relative density of GFP-labeled and tdTomato-labeled axons. The processed images were converted to binary at a threshold cutoff of 4 SDs above mean background pixel intensity, and the quantity of labeled axonal fibers was calculated as the sum of pixels remaining in the three images from each anatomic region. This calculation thus reflects both fiber density and caliber. To quantify the GFP-labeled axons as a percentage of total LC inputs to a region, the number of GFP pixels was divided by the sum of GFP and tdTomato pixels. Fractional innervation by the retrogradely labeled subpopulations across anatomic regions was calculated as the number of GFP pixels in a particular region divided by the number of GFP pixels in all regions imaged (Schwarz et al., 2015). Similarly, fractional innervation by the LC as a whole was calculated as the sum of GFP and tdTomato pixels in a region divided by the sum of GFP and tdTomato pixels in all regions.

\section{Statistical analysis and graphing}

All data are expressed as the mean \pm SEM. Unpaired $t$ tests, two-way ANOVA, and graphing were performed using GraphPad Prism 7 (GraphPad Software Inc.) and Microsoft Excel (Microsoft Corporation). The number of subjects and specific statistical analyses used in each experiment are indicated in the text and figure legends.

\section{Results}

Current viral genetic strategies for mapping efferent projections obtain cellular specificity via injection of two viruses, one into the target region and a second in the cell population of interest, increasing the chance of variable targeting. To remove one of these variables and allow reproducible targeting of genetically defined populations of neurons, we developed the TrAC strategy. TrAC requires three basic elements: (1) a cell-type-specific recombinase driver allele, (2) a dual or triple recombinase-responsive fluorescent indicator allele capable of labeling axons on recombinase activation, and (3) a virus for retrograde delivery of one of the recombinases (Fig. 1). When combining these elements, the virally delivered recombinase is injected into the target region of interest of a transgenic mouse expressing the cell-type-specific recombinase(s) and dual or triple recombinase-responsive fluorescent indicator (Fig. 1). Upon recombination of the indicator allele by the retrogradely delivered recombinase, only genetically defined cells projecting to that target region as well as their axons and collaterals will be marked by the fluorescent reporter (Fig. 1).

To apply TrAC to the LC-NE system, we needed a way to label LC-NE neurons in isolation from other noradrenergic neuron populations. The anatomically defined LC is located in the central gray of the pons adjacent to the lateral edge of the fourth ventricle (Grzanna and Molliver, 1980). Ventrally, the more scattered neurons of the subcoeruleus, separated into dorsal and ventral subdivisions by their position relative to the motor nucleus of the trigeminal, form a continuum between the LC and the more rostral A7 and ventral A5 noradrenergic nuclei (AstonJones, 2004; Paxinos and Franklin, 2013). We exploited the fact that $99.6 \%$ of the anatomically defined LC, together with smaller portions of the dorsal subcoeruleus and A7 immediately adjacent to and continuous with the LC, share a history of the transcription factor engrailed-1 (En1) and the noradrenergic marker dopamine- $\beta$-hydroxylase (Dbh; Robertson et al., 2013). In contrast to viral targeting, use of this genetic classification allows restricted and reproducible targeting of a subpopulation of noradrenergic neurons that closely matches the anatomically defined LC. To label the genetically defined LC-NE neurons in mice, we crossed En $1^{D r e}$ and $D b h^{F / p o}$ to a triple recombinase-responsive indicator (RC::RFLTG; Plummer et al., 2015). In En1 ${ }^{D r e} ; D b h^{\text {Floo; }}$ RC::RFLTG triple transgenic (TrAC-LC) mice, tdTomato expression is restricted to genetically defined LC-NE neurons, switching to EGFP only on retrograde delivery of Cre recombinase (Fig. $2 A)$.

To identify the LC-NE neurons projecting to the mPFC, we injected canine adenovirus encoding Cre recombinase (CAV2-CMV-Cre) into the MPFC of TrAC-LC and Flp-negative littermate control mice and examined fluorescence (Fig. 2A). We found mPFC-projecting NE neurons (EGFP+) intermingled among tdTomato + NE neurons within the LC 
1. Cell type-specific recombinase A driver

2. Dual recombinase-responsive fluorescent indicator

\section{Retrograde virus} (recombinase B)

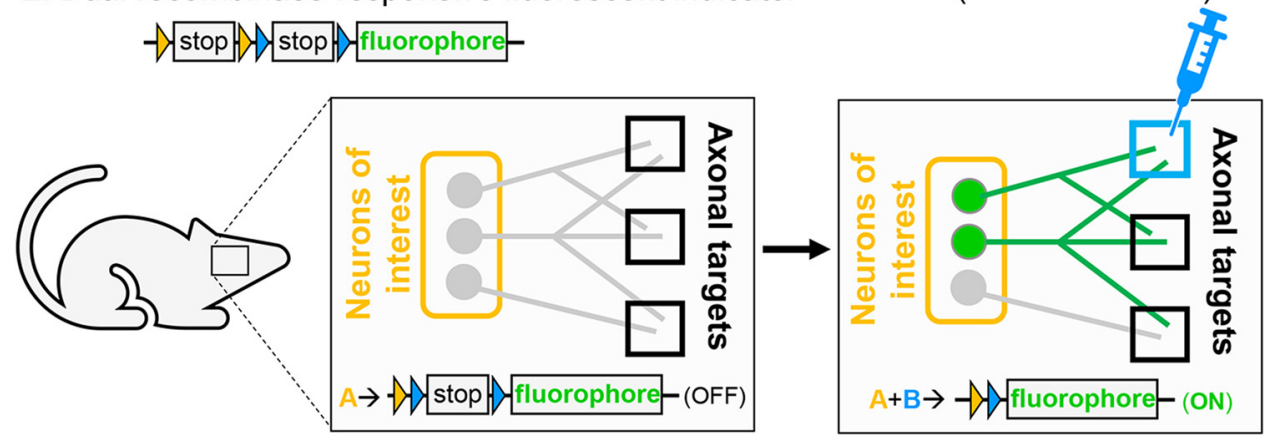

Figure 1. TrAC permits fluorescent labeling of genetically defined neuron populations based on axonal projections. Schematic diagrams show a simple version of TrAC using two recombinases and a dual-recombinase-responsive fluorescent indicator allele. Fluorescent labeling is restricted to neurons expressing recombinase A which project to the brain region injected with the retrograde-transported virus encoding recombinase $\mathrm{B}$.

(Fig. 2B). Ipsilateral to the injection site, we counted an average of $140 \pm 16 \mathrm{mPFC}$-projecting (EGFP + ) and $476 \pm 18$ tdTomato + NE neurons, indicating that CAV2-Cre injection in the mPFC labeled $22.54 \pm 1.9 \%$ of the ipsilateral LC. Contralateral to the injection site, we observed much sparser labeling, with $20 \pm 6$ mPFC-projecting (EGFP + ) NE neurons intermingled among $519 \pm 47$ tdTomato + , indicating that the CAV2-Cre labeled $3.83 \pm 1.3 \%$ of the contralateral LC. Thus, $87.5 \%(140 / 160)$ of the retrogradely labeled neurons were ipsilateral to the injection site, and
$12.5 \%(20 / 160)$ were contralateral to the injection site. This distribution is similar to that reported in previously published reports of LC projections to cortical targets using conventional retrograde tracer techniques (Simpson et al., 1997; Chandler and Waterhouse, 2012; Chandler et al., 2014).

To investigate the distribution of EGFP+ retrogradely labeled mPFC-projecting NE neurons within the LC, we quantified them as a percentage of all labeled neurons (EGFP + tdTomato) in three anatomic domains: the caudal

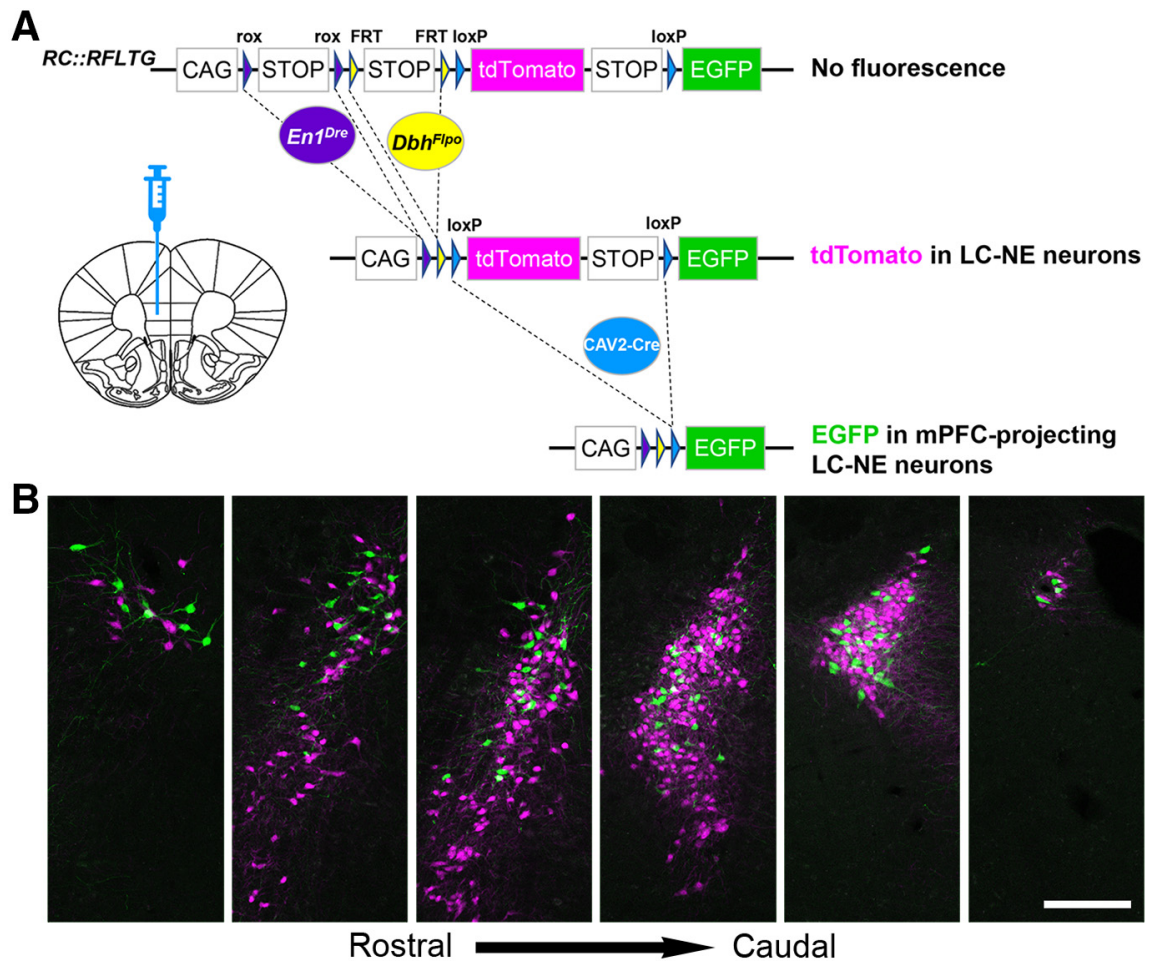

Figure 2. Location of mPFC-protecting noradrenergic neurons within the LC. $\boldsymbol{A}$, Schematic diagram of the $R C$ ::RFLTG indicator allele and coronal schematic of mouse forebrain section showing position of CAV2-Cre injection. $\boldsymbol{B}$, Representative coronal sections through the rostrocaudal extent of the ipsilateral LC from a TrAC-LC mouse (40- $\mu \mathrm{m}$ free-floating sections) showing distribution of EGFP-labeled (green) and tdTomato-labeled (magenta) neurons. Scale bar, $200 \mu \mathrm{m}$. 


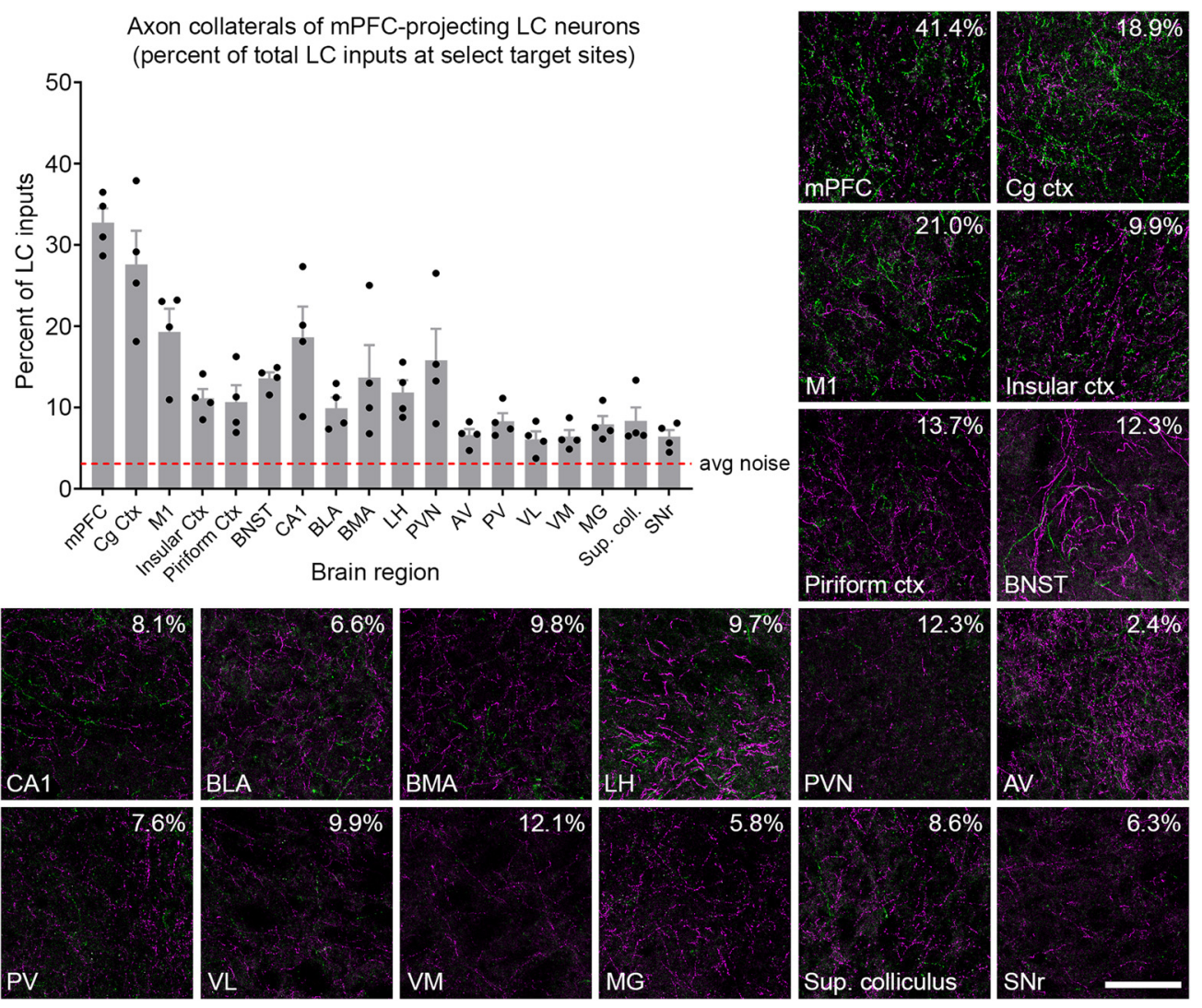

Figure 3. Distribution of axon collaterals from LC neurons projecting to mPFC. Representative fluorescent images show Cre+ (EGFP-labeled, green) and Cre-negative (tdTomato-labeled, magenta) axons in select forebrain and midbrain regions of a TrAC-LC mouse injected with CAV2-Cre in mPFC. Numbers in the upper right corner of each image indicate the percent of LC-NE axons in the image that are EGFP+. The bar graph $(n=4$ mice) indicates percentage of LC-NE axons at each brain region that originate from EGFP + mPFC-projecting LC-NE neurons (mean \pm SEM). The dotted line represents the noise in the green channel (average green pixel count in areas qualitatively lacking EGFP-labeled axons divided by average total pixel count). Percentages below the dotted line are indistinguishable from noise. The scale bar for fluorescent images indicates $100 \mu \mathrm{m}$, and each image represents $13 \%$ of the volume quantified in the bar graph. mPFC, medial prefrontal cortex; Cg Ctx, cingulate cortex; M1, primary motor cortex; BNST, bed nucleus of the stria terminalis; CA1, area CA1 of the hippocampus; BLA, basolateral amygdala; BMA, basomedial amygdala; LH, lateral hypothalamus; PVN, paraventricular hypothalamic nucleus; AV, anteroventral thalamic nucleus; PV, paraventricular thalamic nucleus; VL, ventrolateral thalamic nucleus; VM, ventromedial thalamic nucleus; MG, medial geniculate nucleus; Sup. coll., superior colliculus; SNr, substantia nigra.

core of the anatomically defined LC, the rostral extension of the anatomically defined LC (Grzanna and Molliver 1980), and the subcoeruleus/A7. Ipsilateral to the injection site, EGFP + mPFC-projecting neurons were distributed throughout the rostrocaudal extent of the anatomically defined LC, but they constituted a greater percentage of the rostral extension than the core $(37.13 \pm 4.67 \%$ rostral extension, $22.55 \pm 2.90 \%$ core; $p=0.0378^{a}$, unpaired $t$ test; Table 1). In contrast, EGFP + mPFC-projecting NE neurons were generally absent from the anatomically defined subcoeruleus/A7 (two mice had one EGFP+ neuron). Although there were far fewer EGFP+ neurons in the contralateral anatomically defined LC, compared with the ipsilateral, their rostrocaudal distribution appeared to be the same. As expected, no EGFP+ NE-neurons were observed in Flp-negative littermate control mice.

Next, we analyzed the distribution of axons and collaterals from the retrogradely labeled mPFC-projecting LCNE neurons in select brain regions (Fig. 3). We confirmed noradrenergic identity of the fluorescently labeled axons by immunostaining with an anti-NE transporter (NET) antibody (data not shown). EGFP + collaterals were widespread in multiple cortical regions, including the motor, cingulate, insular, and piriform cortices. In addition, we observed collaterals in multiple subcortical regions, including the bed nucleus of the stria terminalis (BNST), hippocampus, amygdala, hypothalamus, and some thalamic nuclei. Because LC-NE neurons not infected by the CAV2-Cre virus express tdTomato in TrAC-LC mice, we were able to quantify axon collaterals from EGFP+ mPFC-projecting LC-NE neurons as a percentage of all LC-NE inputs to each target region, an analysis that was not possible in prior retrograde viral studies of LC projections (Schwarz et al., 2015; Uematsu et al., 2017). The contribution from EGFP+ neurons was greatest in $\mathrm{mPFC}$ close to the injection site $(32.7 \pm 1.8 \%)$. At other target sites that we examined, the percent contribution from the $\mathrm{EGP}+$ mPFC-projecting LC-NE neurons varied from 


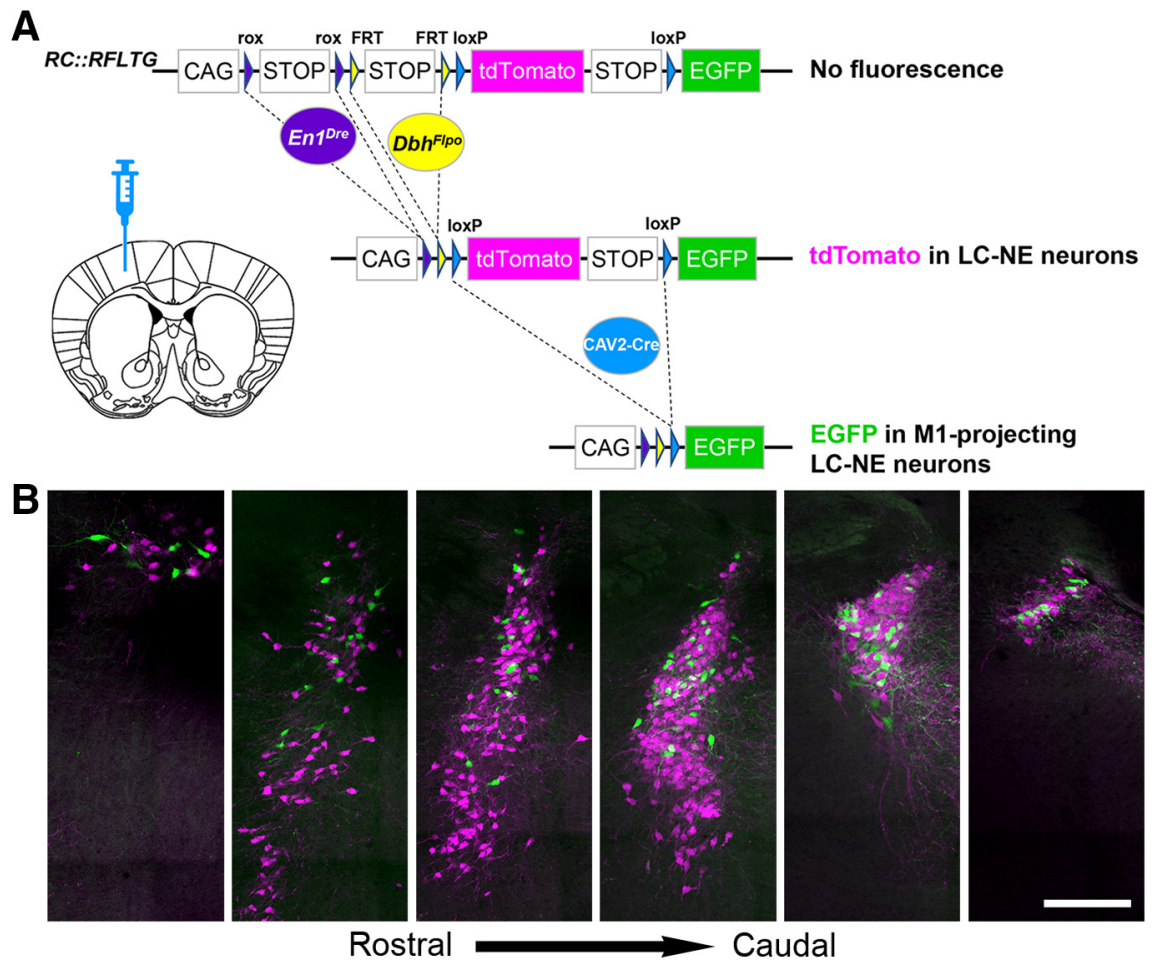

Figure 4. Location of motor cortex-projecting noradrenergic neurons within the LC. $\boldsymbol{A}$, Coronal schematic of mouse forebrain section showing position of CAV2-Cre injection. $\boldsymbol{B}$, Representative coronal sections through the rostrocaudal extent of the ipsilateral LC from a TrAC-LC mouse (40- $\mu \mathrm{m}$ virtual sections from PACT-cleared tissue) showing distribution of EGFP-labeled (green) and tdTomato-labeled (magenta) neurons. Scale bar, $200 \mu \mathrm{m}$.

$27.6 \pm 0.041 \%$ in cingulate cortex ( $\mathrm{Cg}$ ctx) to $6.1 \pm 0.9 \%$ in ventrolateral thalamic nucleus (VL; Fig. 3). Outside the cortex, we observed the highest percent contribution in the hippocampus $(\mathrm{CA} 1 ; 18.6 \pm 3.8 \%)$ and paraventricular hypothalamic nucleus (PVN; $15.8 \pm 3.9 \%$ ). Percent contribution in thalamic nuclei was generally low, but was consistently above the average noise in the images. (Fig. 3).

As with the mPFC injections, CAV2-cre injection in the M1 of TrAC-LC mice labeled a greater percentage of LC-NE neurons on the ipsilateral side. We counted $108 \pm$ 28 M1-projecting (EGFP + ) NE neurons and $571 \pm 42$ tdTomato + NE neurons ipsilateral, and $12 \pm 3 \mathrm{M} 1$-projecting (EGFP+) NE neurons and $617 \pm 55$ tdTomato $+\mathrm{NE}$ neurons contralateral to the injection site. Thus, CAV2Cre injection in M1 labeled $15.19 \pm 3.16 \%$ of the ipsilateral LC and $1.85 \pm 0.39 \%$ of the contralateral. Of the retrogradely labeled neurons in LC, $90 \%(108 / 120)$ were ipsilateral to the M1 injection site and $10 \%(12 / 120)$ were contralateral to the injection site. Again, this distribution is similar to that reported previously for LC projections to cortical targets using conventional retrograde tracer techniques (Simpson et al., 1997; Chandler and Waterhouse, 2012; Chandler et al., 2014). As observed following injection in $\mathrm{mPFC}$, the EGFP-labeled neurons were distributed throughout the rostrocaudal extent of the anatomically defined LC (Fig. 4). Unlike the MPFC-projecting neurons, however, the percentage of M1-projecting (EGFP+) NE neurons was similar in the LC core and rostral extension $(20.03 \pm 4.12 \%$ rostral extension, $19.49 \pm 5.82 \%$ core; $p=0.9418^{\mathrm{b}}$, unpaired $t$ test; Table 1). M1-projecting neurons, like mPFC-projecting neurons, were generally absent from subcoeruleus/A7 (one EGFP+ neuron in one mouse).

When we examined the distribution of axon collaterals from EGFP + M1-projecting LC-NE neurons as a percentage of all LC inputs at target sites (Fig. 5), we again observed the highest percentage of labeled axons near the injection site $(\mathrm{M} 1 ; 33.7 \pm 3.2 \%)$. As with the mPFC injections, we observed widespread EGFP + axon collaterals in cortical and subcortical regions, with percent contributions ranging from $27.2 \pm 1.1 \%$ in $\mathrm{mPFC}$ to $1.4 \pm 0.3 \%$ in anteroventral thalamic nucleus (AV). Outside the cortex, the highest percent contribution of EGFP+ axons was observed in the BNST $(10.3 \pm 0.7 \%)$ and lateral hypothalamus (LH; $9.4 \pm 0.4 \%)$. As with the mPFC-projecting subpopulation, axon collaterals from M1-projecting neurons constituted a small percentage of all LC-NE inputs to thalamic nuclei (Fig. 5). Although contribution from mPFCprojecting neurons was consistently above the average noise (Fig. 3), that from the M1-projecting neurons was at or below the average noise level in all thalamic nuclei that we examined (Fig. 5). This very sparse innervation of the thalamus by the M1-projecting LC subpopulation is notable, given that the LC is the major source of noradrenergic inputs to many thalamic nuclei (Robertson et al., 2013), including several that we examined (e.g., AV, VL, VM). In thalamic regions that receive significant non- $L C$ noradrenergic input (e.g., PV; Robertson et al., 2013), collaterals from the M1-projecting LC subpopulation would represent a particularly small fraction of noradrenergic inputs. 

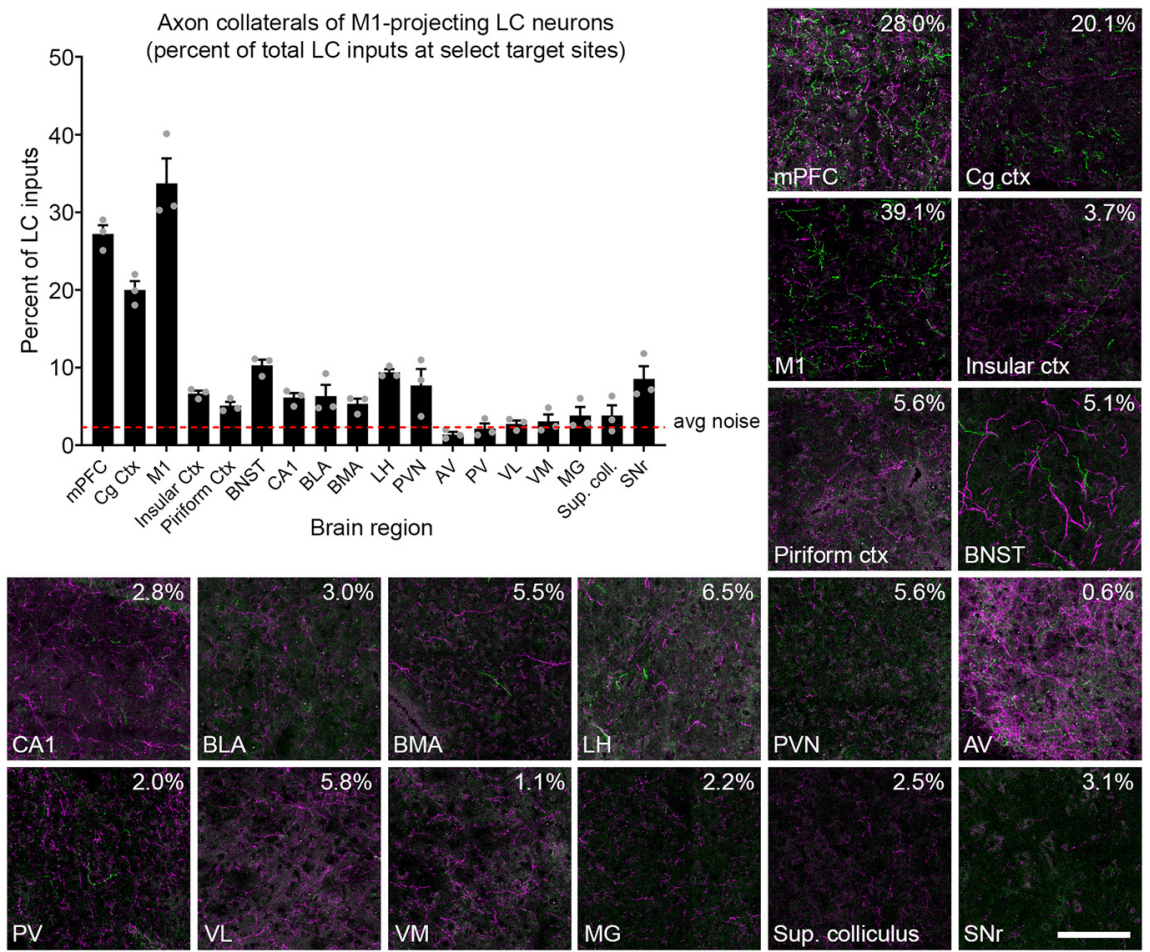

Figure 5. Distribution of axon collaterals from LC neurons projecting to motor cortex. Representative fluorescent images show Cre+ (EGFP-labeled, green) and Cre-negative (tdTomato-labeled, magenta) axons in select forebrain and midbrain regions of a TrAC-LC mouse injected with CAV2-Cre in M1. Numbers in the upper right corner of each image indicate the percent of LC-NE axons in the image that are EGFP + . The bar graph $(n=3$ mice) indicates the percentage of LC-NE axons at each target region that originate from EGFP + M1-projecting LC-NE neurons (are mean \pm SEM). The dotted line represents the noise in the green channel (average green pixel count in areas qualitatively lacking EGFP-labeled axons, divided by average total pixel count). Percentages below the dotted line are indistinguishable from noise. The scale bar for fluorescent images indicates $100 \mu \mathrm{m}$, and each image represents $13 \%$ of the volume quantified in the bar graph.

As an alternative method for analyzing the collateral data, we calculated the fractional distribution of axon collaterals across target regions (i.e., the fraction of a population of axons that innervate each target site). This method has previously been used in retrograde viral analysis of LC projections where the uninfected LC subpopulation was not labeled (Schwarz et al., 2015; Uematsu et al., 2017). Because our analysis, similar to the prior studies, quantifies a fixed volume in brain regions that differ dramatically in size and shape, this analysis reveals relative axon densities, not total axon numbers, in the brain regions. In the present study, with the whole LC labeled, we were able to compare the fractional distribution of projections from each retrogradely labeled subpopulation with that of the LC as a whole (Fig. $6 A, B$ ). Compared with the retrogradely labeled subpopulations, we observed the LC as a whole projecting more uniformly to cortical and subcortical regions, although with a notably large fraction in the anteroventral thalamic nucleus. When we directly compared the distribution of axon collaterals labeled by MPFC or M1 injection (Fig. 6C), we observed the fraction of EGFP + axons to be significantly different at the injection sites $\left(p=0.0217^{\mathrm{C}} \mathrm{mPFC}\right.$ and $p<0.0001^{\mathrm{d}} \mathrm{M} 1$; Table 1$)$. Following injection in $\mathrm{M} 1$, a surprisingly large fraction of EGFP+ axons were observed in MPFC (Fig. 6C), a region which may include both collaterals of M1-projecting neurons and axons of passage projecting to more caudal cortical regions (Morrison et al., 1978, 1979). A greater fraction of efferents from mPFC-projecting LC-NE neurons appear to innervate the thalamus; although the difference did not reach statistical significance in individual regions that were imaged, the sum of fractional innervation of the five thalamic subregions was significantly greater for the mPFC-projecting neurons $(0.1534 \pm 0.0211 \mathrm{mPFC}$, $0.0773 \pm 0.0119 \mathrm{M} 1 ; p=0.0367^{\mathrm{e}}$, unpaired $t$ test; Table 1 ). Taken together, these results suggest that not only are different brain regions (e.g., M1 and $\mathrm{mPFC}$ ) innervated by different subpopulations of LC-NE neurons, but those subpopulations differ from each other and the larger LC$\mathrm{NE}$ system in their pattern of axon collateralization.

\section{Discussion}

The noradrenergic LC represents an extreme example of the ability of projection neurons to form collateral branches. From a small population of neurons, $\sim 2300$ in the bilateral mouse LC (Berger et al., 1979; Touret et al., 1982; Plummer et al., 2017), a dense network of axonal fibers extends throughout the brain and spinal cord. While it is clear that individual LC-NE neurons send collaterals to multiple terminal fields, conventional tracing methods are not capable of identifying the degree or pattern of collateralization in great detail. For example, injecting multiple retrograde tracers into discrete terminal fields to 

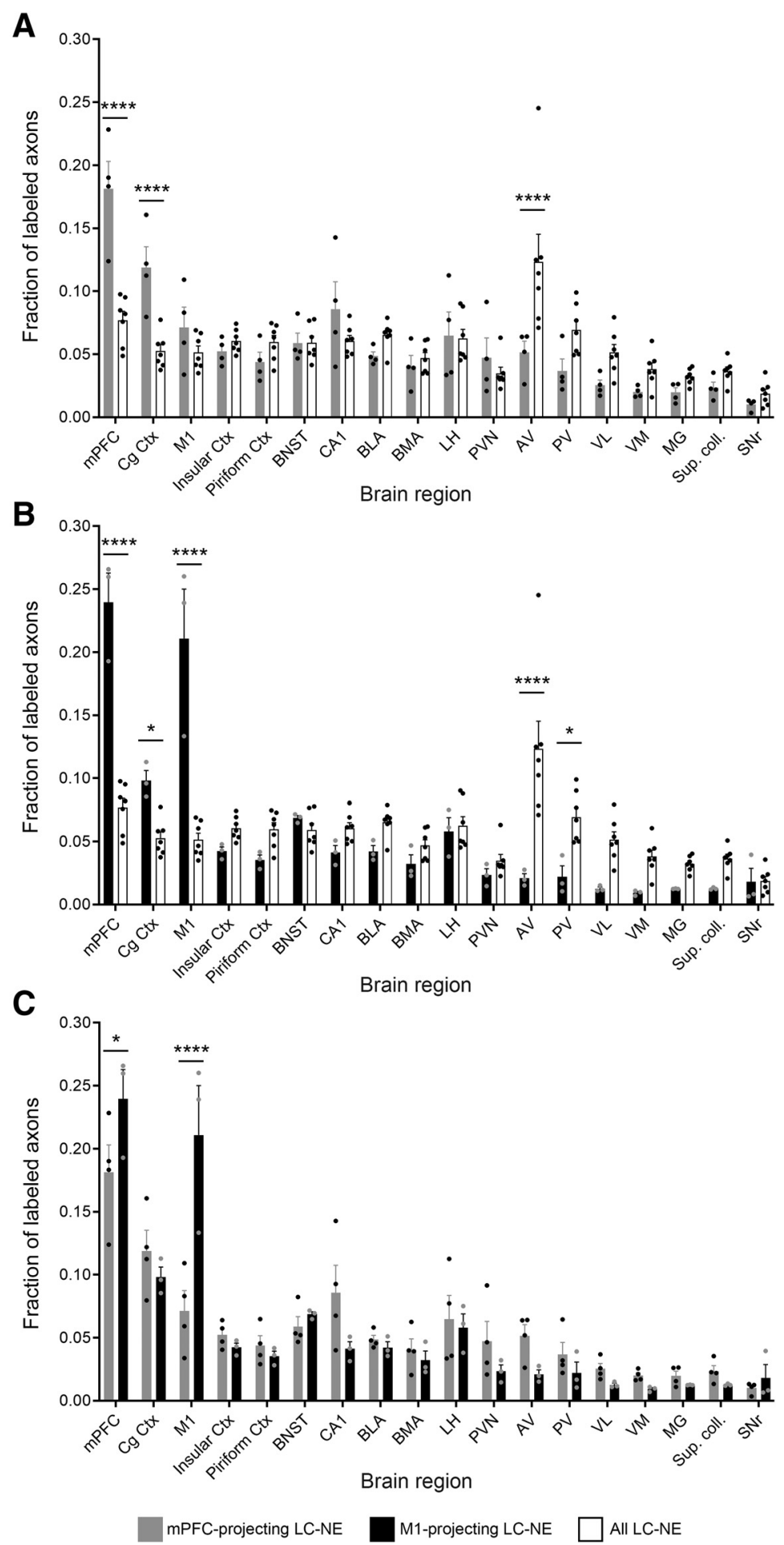

Figure 6. Distribution of axon collaterals from mPFC-projecting and M1-projecting LC-NE neurons differs from that of the LC as a whole and from each other. Bar graphs show fractional distribution of labeled mPFC-projecting, M1-projecting, or all LC-NE neurons at select target regions. Axon density at each region is represented as a fraction of labeled axons in all imaged regions. Bar graph data are mean \pm SEM and were analyzed by two-way repeated measures ANOVA. $\boldsymbol{A}$, Comparison of mPFC-projecting $(n=4$ mice, EGFP + ) and all LC-NE neurons ( $n=7$ mice, sum of EGFP + and tdTomato + axons). LC subpopulation by target region interaction: $F_{(17,153)}=8.072, p<0.0001$. Bonferroni post hoc test, $* * * * p<0.0001[95 \%$ confidence interval of difference (Cl of diff.): mPFC, -0.1441 to $-0.06482 ; \mathrm{Cg}$ Ctx, -0.1059 to -0.02655 ; AV, 0.03218 to 0.1115$]$. $\boldsymbol{B}$, Comparison of M1-projecting ( $n=3$ mice, EGFP+) and all LC-NE neurons (as above). LC subpopulation by target region interaction: $F_{(17,136)}=21.87, p<0.0001$. Bonferroni post hoc test, $* p=0.0176, \mathrm{Cg}$ Ctx $(95 \% \mathrm{Cl}$ of diff.: $-0.08,684$ to -0.004373$)$, $* p=0.0117 \mathrm{PV}$ (95\% Cl of diff.: 0.005982 to 0.08845$)$, $* * * * p<0.0001$ (95\% Cl of diff.: mPFC, -0.2037 to -0.1213 ; M1, -0.2007 to -0.1182 ; AV, 0.06125 to 0.1437 ). C, Comparison of 
continued

mPFC-projecting and M1-projecting LC-NE neurons. Injection site by target region interaction: $F_{(17,85)}=5.152, p<0.0001$. Bonferroni post hoc test, $* p<0.0217$ (95\% Cl of diff.: 0.004639 to 0.1114$)$, $* * * * p<0.0001(95 \% \mathrm{Cl}$ of diff.: 0.08626 to 0.1930$)$.

identify all structures co-innervated by specific subsets of LC cells is impractical since tracer uptake can be unpredictable, as well as too labor intensive for large scale analyses. Recently, two newer strategies have been applied to the LC-NE system. The first, MAPSeq, is a highthroughput sequencing-based approach that provides information about axonal collateralization at single cell resolution, but lacks any information on the spatial organization of LC neurons projecting to a given target or their axonal architecture (Kebschull et al., 2016). The second strategy retains spatial information by employing a dual viral approach to fluorescently label LC neurons projecting to a target brain region. This approach requires retrograde delivery of a recombinase from the target structure and a recombinase-dependent reporter delivered to the LC (Schwarz et al., 2015; Uematsu et al., 2017). The addition of a second viral injection increases the chance of variable targeting, particularly when there is no foolproof method to confirm that the virally delivered reporter is expressed in all LC-NE neurons. While this method can be used successfully to target compact, anatomically discrete structures like the LC, it is of limited value for the study of dispersed cell populations.

TrAC, on the other hand, defines the cell population of interest genetically (without virus) and is not restricted by the distribution of the retrogradely labeled population, making it amenable to the study of dispersed populations. Moreover, the cell population of interest is not limited to cells that are defined by adult gene expression, as is the case with viral labeling. Instead, the population of interest can be defined using transient developmental genetic information. As shown in this article, if the fluorescent indicator allele used in TrAC is capable of simultaneously labeling Cre+ and Cre-negative populations, the retrogradely labeled neurons can be directly observed in the context of the genetically defined population. In the case of complex, heterogeneous neuronal populations such as the central noradrenergic system, use of the triple recombinase responsive allele RC::RFLTG (Plummer et al., 2015) allows analysis to be restricted to a subset of a broader neuronal population. Thus, TrAC allowed us to observe LC axons in isolation at brain targets (e.g., BNST, PVN) that also receive significant inputs from other noradrenergic nuclei. Importantly, this method has allowed us to reliably recapitulate anatomic features that have been previously demonstrated using conventional tracers (e.g., a primarily ipsilateral projection from LC to cortex; Foote et al., 1980; Loughlin et al., 1986; Simpson et al., 1997; Chandler et al., 2014; Figs. 2, 5), while simultaneously revealing previously inaccessible features of axonal collateralization that could not be revealed by tracer molecules.

The data presented in the current study argue for neither restricted and discrete efferent connectivity between LC neurons and their terminal fields (Chandler et al., 2013, 2014), nor for a global broadcast model in which all LC neurons innervate most or all terminal fields (Aston-Jones et al., 1986; Schwarz et al., 2015). Instead, they are broadly consistent with previous analyses (Kebschull et al., 2016; Uematsu et al., 2017) indicating that axons of LC projection neurons tend to have a major terminal field destination which they densely innervate, and widespread minor terminal field targets which contain sparser axonal collaterals than the primary preferred region. Consistent with this model, we found that LC neurons projecting to mPFC have a moderate to sparse network of collaterals to other parts of the cortex and various subcortical locations. While the same generally holds true for cells that project to M1 cortex, one apparent deviation from this trend comes from the observation that an injection of CAV2-CMV-Cre into M1 resulted in a significantly greater fraction of EGFP + axons in MPFC than an injection of the virus into mPFC (Fig. 6B). While this result seems paradoxical, multiple explanations are possible. One possibility is that the labeled axons observed in mPFC after injection in M1 include fibers of passage en route to M1. Another potential explanation, which does not preclude the first, is that LC neurons innervating M1 collateralize to fewer brain regions than do those innervating MPFC, resulting in a larger fraction of the total collaterals from M1projecting neurons localized in a few target regions, with smaller fractions elsewhere. This possibility is supported by the observation that injections into $\mathrm{M} 1$ resulted in a smaller fraction of EGFP+ axons in the thalamus as a whole, relative to injection in $\mathrm{mPFC}$.

A question which arises from this analysis is whether or not the sparser axons that occur outside of the "primary" target do so in some organized fashion with a functional consequence, i.e., could the coordinated release of NE in

Table 1: Statistical table

\begin{tabular}{llll}
\hline & Data structure & Type of test & 95\% confidence interval of difference \\
$\mathrm{a}$ & Normal & Unpaired $t$ test & 1.144 to 28.02 \\
$\mathrm{~b}$ & Normal & Unpaired $t$ test & -16.90 to 17.99 \\
$\mathrm{c}$ & Normal & $\begin{array}{c}\text { Two-way repeated measures ANOVA } \\
\text { (Bonferroni post hoc test) } \\
\text { Two-way repeated measures ANOVA } \\
\text { (Bonferroni post hoc test) }\end{array}$ & 0.004639 to 0.1114 \\
e & Normal & Unpaired $t$ test & 0.08626 to 0.1930 \\
\hline
\end{tabular}

See also Figure 6 legend. 
mPFC and other brain regions by way of axonal collateralization have some operational role in brain function and behavior? It has been previously reported that individual LC neurons collateralize to innervate multiple structures along an ascending sensory pathway (Simpson et al., 1997), possibly as a means of simultaneously promoting NE release throughout a functional network to facilitate sensory encoding. TrAC therefore represents a viable method of identifying networks among which release of NE or other LC co-transmitters might occur simultaneously to facilitate a specific neurophysiological or behavioral functional role. The functional organization of LC-NE axon collaterals to cognitive, sensory and motor terminal fields can now be probed using this approach.

The difference in the rostrocaudal distribution of mPFCprojecting and M1-projecting neurons suggests that they constitute distinct, although overlapping, subpopulations of LC-NE neurons. However, our current inability to simultaneously label neurons projecting to two different injection sites precludes determination of the precise degree of overlap between the two subpopulations. Such analysis may become possible with a new generation of tools, including CAV2 vectors expressing recombinases other than Cre and indicator alleles like RC::RFLTG that express different fluorescent proteins in response to combinatorial recombinase expression. Nevertheless, the current TrAC strategy, by simultaneously labeling a genetically defined neuronal population and a projection-based subpopulation, permits analyses of axon collateralization that have not previously been practicable in complex genetically and neurochemically defined neuronal subtypes.

\section{References}

Aston-Jones G (2004) Locus coeruleus, A5 and A7 noradrenergic cell groups. In: The rat nervous system (Paxinos G, ed), pp 253294. San Diego: Academic Press.

Aston-Jones G, Ennis M, Pieribone VA, Nickell WT, Shipley MT (1986) The brain nucleus locus coeruleus: restricted afferent control of a broad efferent network. Science 234:734-737.

Bellesi M, Tononi G, Cirelli C, Serra PA (2016) Region-specific dissociation between cortical noradrenaline levels and the sleep/wake cycle. Sleep 39:143-154.

Berger B, Hervé D, Dolphin A, Barthelemy C, Gay M, Tassin JP (1979) Genetically determined differences in noradrenergic input to the brain cortex: a histochemical and biochemical study in two inbred strains of mice. Neuroscience 4:877-888.

Bloom FE, Hoffer BJ, Siggins GR (1971) Studies on norepinephrinecontaining afferents to Purkinje cells of rat cerebellum. I. Localization of the fibers and their synapses. Brain Res 25:501521.

Chandler D, Waterhouse BD (2012) Evidence for broad versus segregated projections from cholinergic and noradrenergic nuclei to functionally and anatomically discrete subregions of prefrontal cortex. Front Behav Neurosci 6:20.

Chandler DJ, Lamperski CS, Waterhouse BD (2013) Identification and distribution of projections from monoaminergic and cholinergic nuclei to functionally differentiated subregions of prefrontal cortex. Brain Res 1522:38-58.

Chandler DJ, Gao WJ, Waterhouse BD (2014) Heterogeneous organization of the locus coeruleus projections to prefrontal and motor cortices. Proc Natl Acad Sci USA 111:6816-6821.

Chandler DJ, Jensen P, McCall JG, Pickering AE, Schwarz LA, Totah NK (2019) Redefining noradrenergic neuromodulation of behavior: impacts of a modular locus coeruleus architecture. J Neurosci 39:8239-8249.

Foote SL, Loughlin SE, Cohen PS, Bloom FE, Livingston RB (1980) Accurate three-dimensional reconstruction of neuronal distributions in brain: reconstruction of the rat nucleus locus coeruleus. $J$ Neurosci Methods 3:159-173.

Foote SL, Bloom FE, Aston-Jones G (1983) Nucleus locus ceruleus: new evidence of anatomical and physiological specificity. Physiol Rev 63:844-914.

Grzanna R, Molliver ME (1980) The locus coeruleus in the rat: an immunohistochemical delineation. Neuroscience 5:21-40.

Hirschberg S, Li Y, Randall A, Kremer EJ, Pickering AE (2017) Functional dichotomy in spinal- vs prefrontal-projecting locus coeruleus modules splits descending noradrenergic analgesia from ascending aversion and anxiety in rats. Elife 6:e29808.

Hnasko TS, Perez FA, Scouras AD, Stoll EA, Gale SD, Luquet S, Phillips PE, Kremer EJ, Palmiter RD (2006) Cre recombinase-mediated restoration of nigrostriatal dopamine in dopamine-deficient mice reverses hypophagia and bradykinesia. Proc Natl Acad Sci USA 103:8858-8863.

Howorth PW, Teschemacher AG, Pickering AE (2009) Retrograde adenoviral vector targeting of nociresponsive pontospinal noradrenergic neurons in the rat in vivo. J Comp Neurol 512:141-157.

Kebschull JM, Garcia da Silva P, Reid AP, Peikon ID, Albeanu DF, Zador AM (2016) High-throughput mapping of single-neuron projections by sequencing of barcoded RNA. Neuron 91:975-987.

Li Y, Hickey L, Perrins R, Werlen E, Patel AA, Hirschberg S, Jones MW, Salinas S, Kremer EJ, Pickering AE (2016) Retrograde optogenetic characterization of the pontospinal module of the locus coeruleus with a canine adenoviral vector. Brain Res 1641:274290.

Loughlin SE, Foote SL, Bloom FE (1986) Efferent projections of nucleus locus coeruleus: topographic organization of cells of origin demonstrated by three-dimensional reconstruction. Neuroscience 18:291-306.

Matthies HJ, Han Q, Shields A, Wright J, Moore JL, Winder DG, Galli A, Blakely RD (2009) Subcellular localization of the antidepressant-sensitive norepinephrine transporter. BMC Neurosci 10:65.

McCall JG, Siuda ER, Bhatti DL, Lawson LA, McElligott ZA, Stuber GD, Bruchas MR (2017) Locus coeruleus to basolateral amygdala noradrenergic projections promote anxiety-like behavior. Elife 6: e18247.

Morrison JH, Grzanna R, Molliver ME, Coyle JT (1978) The distribution and orientation of noradrenergic fibers in neocortex of the rat: an immunofluorescence study. J Comp Neurol 181:17-39.

Morrison JH, Molliver ME, Grzanna R (1979) Noradrenergic innervation of cerebral cortex: widespread effects of local cortical lesions. Science 205:313-316.

Paxinos G, Franklin KBJ (2013) The mouse brain in stereotaxic coordinates. San Diego: Academic Press.

Plummer NW, Evsyukova IY, Robertson SD, de Marchena J, Tucker CJ, Jensen P (2015) Expanding the power of recombinase-based labeling to uncover cellular diversity. Development 142:43854393.

Plummer NW, de Marchena J, Jensen P (2016) A knock-in allele of En1 expressing dre recombinase. Genesis 54:447-454.

Plummer NW, Scappini EL, Smith KG, Tucker CJ, Jensen P (2017) Two subpopulations of noradrenergic neurons in the locus coeruleus complex distinguished by expression of the dorsal neural tube marker Pax7. Front Neuroanat 11:60.

Powell JM, Plummer NW, Scappini EL, Tucker CJ, Jensen P (2019) DEFiNE: a method for enhancement and quantification of fluorescently labeled axons. Front Neuroanat 12:117.

Robertson SD, Plummer NW, de Marchena J, Jensen P (2013) Developmental origins of central norepinephrine neuron diversity. Nat Neurosci 16:1016-1023.

Schindelin J, Arganda-Carreras I, Frise E, Kaynig V, Longair M, Pietzsch T, Preibisch S, Rueden C, Saalfeld S, Schmid B, Tinevez JY, White DJ, Hartenstein V, Eliceiri K, Tomancak P, Cardona A 
(2012) FIJI: an open-source platform for biological-image analysis. Nat Methods 9:676-682.

Schwarz LA, Miyamichi K, Gao XJ, Beier KT, Weissbourd B, DeLoach KE, Ren J, Ibanes S, Malenka RC, Kremer EJ, Luo L (2015) Viral-genetic tracing of the input-output organization of a central noradrenaline circuit. Nature 524:88-92.

Simpson KL, Altman DW, Wang L, Kirifides ML, Lin RC, Waterhouse BD (1997) Lateralization and functional organization of the locus coeruleus projection to the trigeminal somatosensory pathway in rat. J Comp Neurol 385:135-147.

Touret M, Valatx JL, Jouvet M (1982) The locus coeruleus: a quantitative and genetic study in mice. Brain Res 250:353-357.
Uematsu A, Tan BZ, Johansen JP (2015) Projection specificity in heterogeneous locus coeruleus cell populations: implications for learning and memory. Learn Mem 22:444-451.

Uematsu A, Tan BZ, Ycu EA, Cuevas JS, Koivumaa J, Junyent F, Kremer EJ, Witten IB, Deisseroth K, Johansen JP (2017) Modular organization of the brainstem noradrenaline system coordinates opposing learning states. Nat Neurosci 20:16021611.

Yang B, Treweek JB, Kulkarni RP, Deverman BE, Chen CK, Lubeck E, Shah S, Cai L, Gradinaru V (2014) Single-cell phenotyping within transparent intact tissue through whole-body clearing. Cell 158:945-958. 